\title{
Protective effects of curcumin against neuroinflammation induced by Aß25-35 in primary rat microglia: modulation of high-mobility group box 1, toll-like receptor 4 and receptor for advanced glycation end products expression
}

\author{
Wei He", Kaiming Yuan", Bin Ji, Yuan Han, Jun Li \\ Department of Anesthesiology and Perioperative Medicine, Critical Care and Pain Medicine, The Second Affiliated Hospital \& Yuying Children \\ Hospital of Wenzhou Medical University, Wenzhou 325027, China \\ Contributions: (I) Conception and design: J Li, Wei He; (II) Administrative support: K Yuan; (III) Provision of study materials and patients: W He, \\ Y Han ; (IV) Collection and assembly of data: B Ji; (V) Data analysis and interpretation: W He; (VI) Manuscript writing: All authors; (VII) Final \\ approval of manuscript: All authors. \\ "These authors contributed equally to this work. \\ Correspondence to: Dr. Jun Li. Department of Anesthesiology and Perioperative Medicine, Critical Care and Pain Medicine, The Second Affiliated \\ Hospital \& Yuying Children Hospital of Wenzhou Medical University, West College Road 109, Wenzhou 325027, China. Email: lijun0068@163.com.
}

\begin{abstract}
Background: Activated microglia induced by amyloid-beta $(\mathrm{A} \beta)$ release proinflammatory cytokines that can induce neurotoxicity. High-mobility group box 1 protein (HMGB1) and HMGB1-mediated inflammatory responses have been attributed with memory impairment in patients with Alzheimer's disease (AD). There is accumulating evidence to suggest curcumin is a potent anti-inflammatory polyphenol. However, whether curcumin could effectively inhibit inflammation through the suppression of HMGB1 production or HMGB1-mediated inflammatory responses in A $\beta$-activated microglia is still unclear.

Methods: Primary microglia were prepared from the cerebral cortices of one- to three-day-old Sprague Dawley rats. The microglia were cultured and treated with $A \beta_{25-35} 50 \mu M$ for $24 \mathrm{~h}$ to prove a toxic effect. Curcumin $10 \mu \mathrm{M}$ was administrated $1 \mathrm{~h}$ before $A \beta_{25-35}$ treatment. The levels of HMGB1, interleukin-1 $\beta$ (IL$1 \beta)$, and tumor necrosis factor- $\alpha(\mathrm{TNF}-\alpha)$ in the culture medium were analyzed by ELISA. Western blotting was conducted to assess the expression level of toll-like receptor 4 (TLR4) and the receptor for advanced glycation end products (RAGE). In addition, PC12 cells were treated with conditioned medium from microglia treated with $A \beta_{25-35}$ or $A \beta_{25-35}$ and curcumin, and cell viability was subsequently assessed by MTT.

Results: Curcumin was found to significantly inhibit HMGB1 expression and release in $A \beta_{25-35}$-stimulated microglia. Pretreatment with curcumin reduced TLR4 and RAGE expression. Proinflammatory cytokines such as IL-1 $\beta$ and TNF- $\alpha$ were also remarkably reduced by curcumin. In addition, curcumin protected neurons from indirect toxicity mediated by $\mathrm{A} \beta_{25-35}$-treated microglia.

Conclusions: Curcumin effectively inhibits $A \beta_{25-35}$-induced neuroinflammation in microglia, partly by suppressing the expression of HMGB1, TLR4, and RAGE.
\end{abstract}

Keywords: Curcumin; amyloid-beta (A $\beta$ ); microglia; high mobility group box-1 protein 1 (HMGB1)

Submitted Nov 25, 2019. Accepted for publication Dec 13, 2019.

doi: $10.21037 /$ atm.2019.12.147

View this article at: http://dx.doi.org/10.21037/atm.2019.12.147 


\section{Introduction}

Alzheimer's disease (AD) is a progressive neurodegenerative disorder characterized by intracellular neurofibrillary tangles and extracellular deposition of amyloid-beta (A $\beta)(1)$. Microglia, the resident immune cells in the brain, are the first line of defense to protect the CNS from injury and invading pathogens (2). Activated microglia is induced by $\mathrm{A} \beta$ release of several proinflammatory mediators and can induce neurotoxicity and promote the pathological cascade of $\mathrm{AD}(3,4)$.

The high mobility group box-1 protein 1 (HMGB1), a non-histone chromosomal binding protein released from damaged cells or cells under stressful conditions, can directly induce tissue damage and promote the release of proinflammatory mediators $(5,6)$. HMGB1mediated inflammatory responses through receptor for advanced glycation end products (RAGE) and toll-like receptor 4 (TLR4), which have been implicated in memory impairments in $\mathrm{AD}$ (7-9). Preventing the production of HMGB1 or HMGB1-mediated inflammatory responses may function as a therapeutic target for $\mathrm{AD}$.

Curcumin, a yellow-colored ingredient of the spice turmeric, was extracted from the rhizome of curcuma longa (turmeric). Curcumin has powerful antioxidant, anti-inflammatory and anti-amyloidogenic properties that may have neuroprotective effects in AD (10-13). Also, recent studies have suggested that curcumin protects against propionibacterium acne (14) or concanavalin A-induced liver injury $(15,16)$ through inhibition of HMGB1 cytoplasmic translocation and expression. Besides, curcumin could decrease the levels of HMGB1, TLR4 or TLR2 expression in fibrogenesis hepatocytes (17) and lipopolysaccharide-treated human endothelial cells (18). Whether curcumin could be an effective agent for inhibiting inflammation by suppressing the production of HMGB1 or HMGB1-mediated inflammatory responses in $A \beta$-activated microglia is still unclear.

In this study, we examined whether curcumin inhibits $A \beta 25$ 35 -induced neuroinflammation in primary rat microglia by suppressing HMGB1, TLR4, and RAGE expression.

\section{Methods}

All animal experiments were conducted following the animal protocols approved by the Animal Ethics Committee of the Second Affiliated Hospital \& Yuying Children Hospital of Wenzhou Medical University.

\section{Chemicals}

3-(4,5-dimethylthiazol-2-yl)-2,5-diphenyltetrazolium bromide (MTT), dimethyl sulfoxide (DMSO), A $325-35$, and curcumin were purchased from Sigma-Aldrich (St. Louis, MO, USA). Rabbit polyclonal anti-TLR4, anti-RAGE antibodies, and rabbit monoclonal anti-GAPDH antibody were from Cell Signaling Technology (Beverly, USA). Rabbit monoclonal anti-HMGB1 antibody was from Epitomics, Inc. (Burlingame, CA, USA). Horseradish peroxidase (HRP)-conjugated goat-anti-rabbit secondary antibody was purchased from Kangcheng Biotech Co. Ltd. (Shanghai, China). Tumor necrosis factor- $\alpha$ (TNF- $\alpha$ ) and interleukin- $1 \beta$ (IL-1 $\beta$ ) ELISA kits were from R\&D Systems (Minneapolis, MN, USA). High mobility group protein B1 (HMGB1) ELISA kit was from Shino-Test Co., (Kyoto, Japan).

\section{Preparation of Aß25-35}

A 25-35 was dissolved in DMSO at $10 \mathrm{mM}$ and stored at $-20{ }^{\circ} \mathrm{C}$ until use. The stock solution was diluted with deionized distilled water to a proper concentration and incubated at $37{ }^{\circ} \mathrm{C}$ for 4 days for aggregation before use. The final concentration of DMSO was less than $0.01 \%$, which did not affect cell viability.

\section{Aß25-35 treatment and curcumin administration}

Microglia were treated with A $325-3550 \mu \mathrm{M}$ for $24 \mathrm{~h}$ to prove a toxic effect. Curcumin $10 \mu \mathrm{M}$ was administrated $1 \mathrm{~h}$ before A $\beta 25-35$ treatment.

\section{Cell culture}

Sprague Dawley rats were purchased from the Experimental Animal Center of Wenzhou University. As previously described (19), primary microglia were prepared from the cerebral cortices of 1- to 3-day-old Sprague Dawley rats. Briefly, cortical tissue was triturated into single cells and incubated in DMEM holding 10\% FBS. After centrifugation, cells were suspended and plated in poly-D-lysine coated $75 \mathrm{~cm}^{2}$ flasks $\left(1 \times 10^{7}\right.$ cells/flask) for $9-10$ days at $37^{\circ} \mathrm{C}$ in a humidified $95 \%$ air and $5 \% \mathrm{CO}_{2}$ incubator. Microglia were then detached from the flasks by mild shaking for $2 \mathrm{~h}$ at $200 \mathrm{rpm}$. Reserved supernatant was harvested and filtered through a nylon mesh to remove astrocytes. Cells were plated in six-well plates $\left(2 \times 10^{6}\right.$ cells/well) for $1 \mathrm{~h}$ and were washed to remove unattached cells before use. 

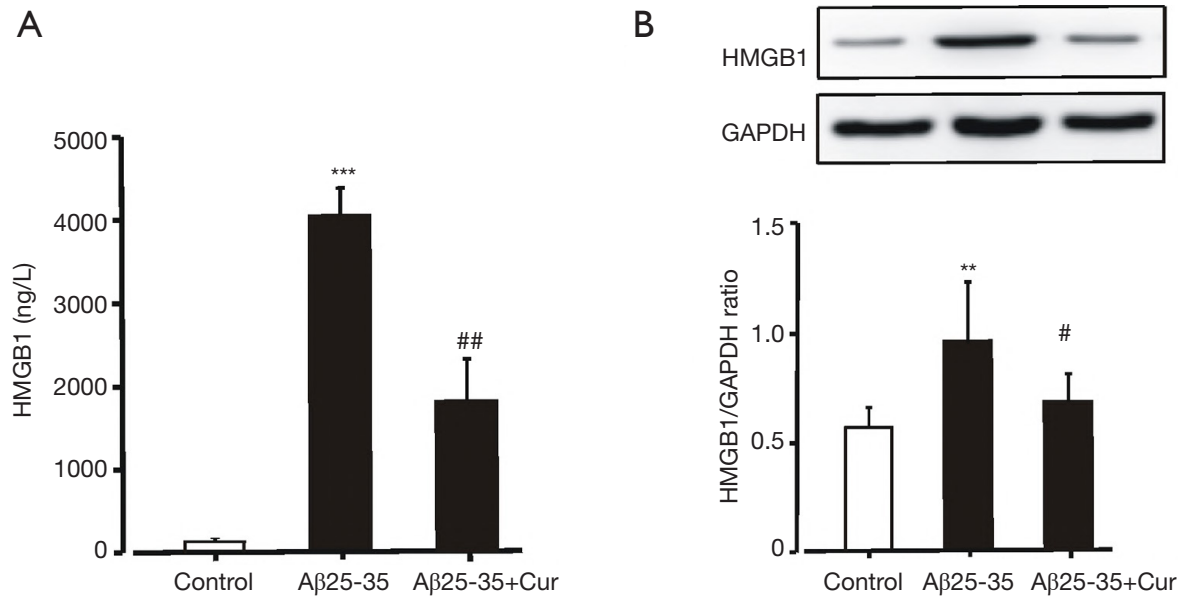

Figure 1 Effects of curcumin on HMGB1 production in A 325 -35-stimulated microglia. Microglia were pretreated with or without curcumin $(10 \mu \mathrm{M})$ for $1 \mathrm{~h}$ before A $\beta 25-35(50 \mu \mathrm{M})$ incubation for $24 \mathrm{~h}$. (A) The concentration of HMGB1 in the culture medium was analyzed by ELISA. ***, $\mathrm{P}<0.001$, vs. control; \#\#, $\mathrm{P}<0.01$, vs. A $\beta 25-35$. (B) HMGB1 expression was examined by western blot. **, $\mathrm{P}<0.01$, vs. control; \#, $\mathrm{P}<0.05$, vs. A $325-35$. Microglia treated with PBS were set as control. All data were expressed as mean \pm standard deviation ( $\mathrm{n}=8$ ). Cur, curcumin; HMGB1, high mobility group box-1 protein 1; A $\beta$, amyloid-beta; ELISA, enzyme-linked immunosorbent assay; PBS, phosphate buffer saline.

Pheochromocytoma (PC12) cells were obtained from the Institute of Biochemistry and Cell Biology, SIBS, CAS (Shanghai, China). PC12 Cells were planted in $25 \mathrm{~cm}^{2}$ flasks and incubated in DMEM holding $10 \% \mathrm{FBS}$ at $37^{\circ} \mathrm{C}$ in a humidified $95 \%$ air and $5 \% \mathrm{CO}_{2}$ incubator.

\section{Cell viability assay}

Cell viability was assessed by MTT assay as described previously (20). In brief, PC12 Cells were seeded in 96 well plates at a density of $5 \times 10^{3}$ cells/well in serum-free DMEM and allowed to adhere at $37^{\circ} \mathrm{C}$ for $24 \mathrm{~h}$. After treatment with a conditioned medium for $24 \mathrm{~h}, 20 \mu \mathrm{L}$ MTT reagent (final concentration, $2.5 \mathrm{mg} / \mathrm{mL}$ ) was added to each well and the plate was incubated at $37^{\circ} \mathrm{C}$ for $4 \mathrm{~h}$. The medium was aspirated off, and $150 \mu \mathrm{L}$ DMSO was added to each well. The optical density of each well was read at $570 \mathrm{~nm}$ using a microplate reader (ELX808, Biotek, Winooski, VT, USA).

\section{Western blot analysis}

Proteins were loaded and separated in 10\% SDS-PAGE and transferred to PVDF membranes. The membranes were blocked with $5 \%$ non-fat milk for $2 \mathrm{~h}$ at room temperature and followed by incubation with primary antibodies overnight at $4{ }^{\circ} \mathrm{C}$. The membranes were then incubated with the HRP-conjugated secondary antibodies for $2 \mathrm{~h}$ at room temperature, and the expression of the protein was visualized by the enhanced chemiluminescence reagent (ECL) using an Imaging System (ChemiDoc MP, Bio-Rad).

\section{Enzyme-linked immunosorbent assay (ELISA)}

The levels of TNF- $\alpha$ IL-1 $\beta$ (R\&D Systems, Minneapolis, MN, USA), and HMGB1 (Shino-Test Co., Kyoto, Japan) in the culture supernatants were measured with ELISA kits according to the manufacturer's instructions.

\section{Statistical analysis}

Results were presented as mean \pm standard deviation. Statistical analyses were performed using SPSS 16.0 (SPSS, Chicago, IL, USA). Differences among experimental groups were evaluated by one-way analysis of variance (ANOVA). $\mathrm{P}<0.05$ was considered statistically significant.

\section{Results}

\section{Curcumin inbibits HMGB1 production in Aß25-35- stimulated microglia}

We supposed that curcumin could be an effective agent for inhibiting neuroinflammation by suppressing the production 
of HMGB1. Our preliminary results showed that the concentration of HMGB1 in the culture medium increased significantly after the A $325-35$ stimulation. Pretreatment with curcumin significantly decreased the concentration of HMGB1 in the culture medium (Figure 1A). Curcumin also significantly inhibited HMGB1 expression in microglia treated with A $325-35$ (Figure 1B). These results suggest that curcumin inhibits HMGB1 production in A $\beta 25-35$ stimulated microglia.

\section{Curcumin inbibits HMGB1-mediated inflammatory responses in Aß25-35-stimulated microglia}

HMGB1-mediated inflammatory responses through RAGE and TLR4 had been implicated in memory impairments and cell death in $\mathrm{AD}$ (7-9). To explore whether curcumin inhibits HMGB1-mediated inflammatory responses in A 225-35-stimulated microglia, we first examined the effects of curcumin on the expressions of RAGE and TLR4. A 325 35 induced a significantly increased expression of RAGE and TLR4 (Figure 2A,B). Pretreated with curcumin could significantly inhibit RAGE and TLR4 expression. The levels of IL- $1 \beta$ and TNF- $\alpha$ in the culture medium were significantly reduced by curcumin (Figure 2C,D). These results suggest that curcumin inhibits HMGB1-mediated inflammatory responses in A $\beta 25$-35-stimulated microglia by decreasing the expression of RAGE and TLR4.

\section{Curcumin inbibits the toxicity of AB25-35-activated microglia to neurons}

Because our results suggest that curcumin inhibits HMGB1 production and HMGB1-mediated inflammatory responses in A 325 -35-stimulated microglia. Next, we investigated whether curcumin inhibits the toxicity of activated microglia to neurons. We used an MTT assay to examine the effect of curcumin on the toxicity of A 25 -35-activated microglia PC12 cells. The cell viability was significantly decreased after treatment with conditioned medium from A $325-35$ treated microglia. However, when PC12 cells were incubated in conditioned medium from microglia treated with curcumin and A 25 -35, the cell viability was significantly increased (Figure 3). Our data suggest that curcumin inhibits the toxicity of A $325-35$-activated microglia to neurons.

\section{Discussion}

Because of its potent antioxidant, anti-inflammatory and anti-amyloidogenic properties, curcumin, a natural ingredient extracted from Curcuma longa, has shown potential therapeutic effect for AD (10-13). HMGB1 can directly induce neuronal damage and promote the release of inflammatory mediators, which is a risk factor for $\mathrm{AD}(5-9)$, as a non-histone chromosomal binding protein released from damaged cells or cells under stressful conditions.

Takata $e t$ al. found that the level of HMGB1 was significantly increased, and HMGB1 expression was colocalized in senile plaques associated with microglia in the tested $\mathrm{AD}$ rat brain samples (21). They further performed another two studies and found that extracellular HMGB1 released from dying neurons could decrease microglial $A \beta$ clearance, which enhanced the neurotoxicity of $A \beta(22,23)$. Thus, the inhibition of HMGB1 production may be a therapeutic strategy for AD. However, so far, the effect of curcumin on HMGB1 expression in $A \beta$-activated microglia is still unclear.

Recent studies have indicated that curcumin could be an effective agent for inhibiting the HMGB1 cytoplasmic translocation and expression in the acute inflammatory liver injury model (14-16). Two earlier studies had shown the effects of curcumin pretreatment on concanavalin A-induced hepatitis in mice. Curcumin significantly suppressed the intrahepatic expression of HMGB1 by inhibiting the translocation from the nucleus to the cytoplasm $(15,16)$. Gu et al. also found that curcumin treatment attenuated propionibacterium acnes-induced liver injury through reducing cytoplasmic expression of HMGB1 (14). Thus, we suspected that curcumin pretreatment could reduce the expression of HMGB1 in A 25 -35-stimulated microglia. Indeed, our results showed that curcumin could significantly reduce the HMGB1 level in the culture medium and decrease the expression of HMGB1 in microglia stimulated with $\mathrm{A} \beta 25-35$.

Once released from the cytoplasm, extracellular HMGB1 can induce a pro-inflammatory response through binding to RAGE and TLR4 (7-9). Several recent studies had examined these effects of curcumin on HMGB1-mediated proinflammatory responses. In HMGB1-activated human umbilical endothelial cells, curcumin not only decreased the cell surface expression of TLR2 and TLR4 but also inhibited the levels of VCAM-1, ICAM-1, and E-selectin (18). $\mathrm{Gu} e t \mathrm{al}$. found that propionibacterium acne treatment can increase the expression of TLR2, TLR4, and RAGE in mice liver. Curcumin treatment significantly reduced the expression of TLR4, but not of RAGE and TLR2 (14). However, the effects of curcumin on HMGB1-mediated proinflammatory responses have not been explored in Aß25- 
A
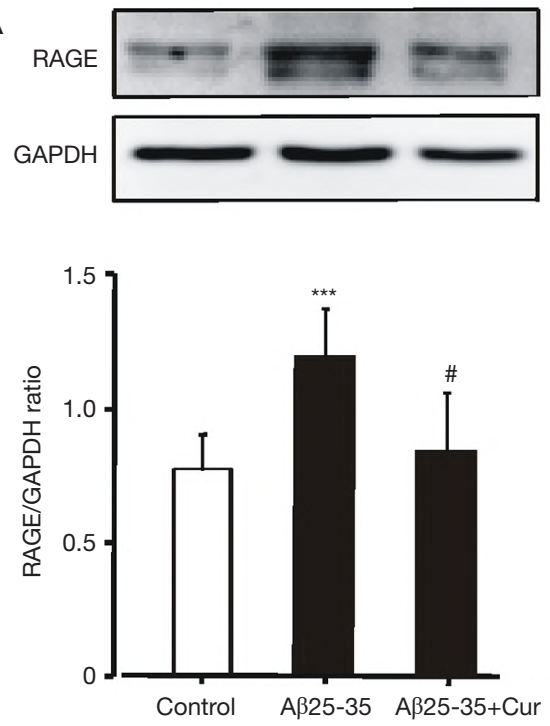

C

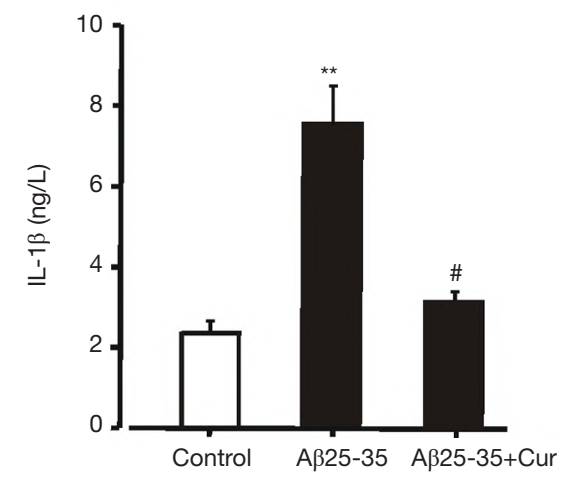

B
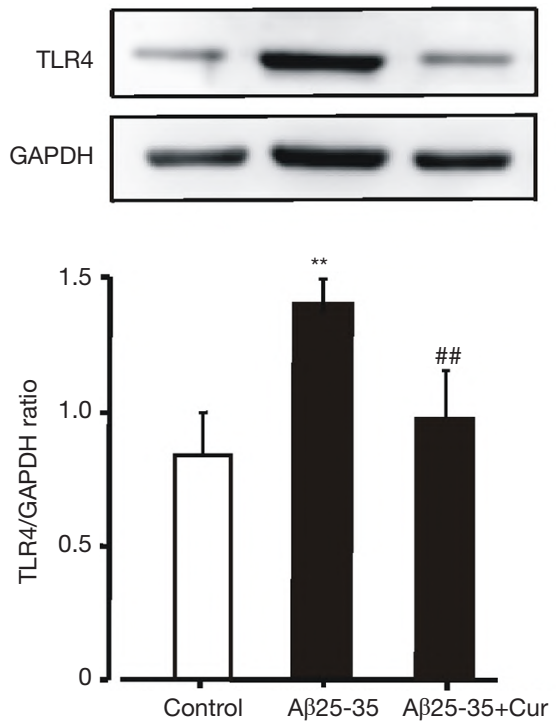

D

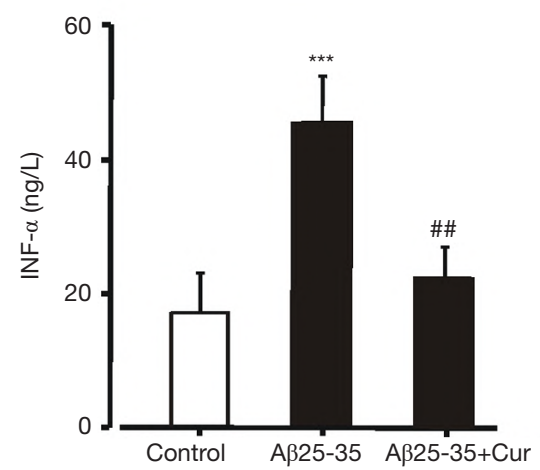

Figure 2 Effects of curcumin on HMGB1-mediated inflammatory responses in Aß25-35-stimulated microglia. Microglia were pretreated with or without curcumin $(10 \mu \mathrm{M})$ for $1 \mathrm{~h}$ before AB25-35 (50 $\mu \mathrm{M})$ incubation for $24 \mathrm{~h}$. The expressions of RAGE (A) and TLR4 (B) were assessed by western blot. The levels of IL- $1 \beta(\mathrm{C})$ and TNF- $\alpha(\mathrm{D})$ in the culture medium were analyzed by ELISA. ${ }^{* *}, \mathrm{P}<0.001 ;{ }^{* *}, \mathrm{P}<0.01$, vs. control; \#\#, $\mathrm{P}<0.01$; \#, $\mathrm{P}<0.05$, vs. A 25 -35. Microglia treated with PBS were set as control. All data were expressed as mean \pm standard deviation ( $\mathrm{n}=8$ ). Cur, curcumin; HMGB1, high mobility group box-1 protein 1; A $\beta$, amyloid-beta; TLR4, toll-like receptor 4; RAGE, receptor for advanced glycation end products; ELISA, enzyme-linked immunosorbent assay; TNF- $\alpha$, tumor necrosis factor- $\alpha$; IL- $1 \beta$, interleukin-1 $\beta$; PBS, phosphate buffer saline.

35 -stimulated microglia. In the present study, we found that A 25 -35 induced significant increased expression of TLR4 and RAGE in microglia. Pretreated with curcumin could significantly inhibit the expression of TLR4 and RAGE. The proinflammatory cytokines such as TNF- $\alpha$ and IL$1 \beta$ expression were also remarkably reduced by curcumin. These results indicated that curcumin inhibits HMGB1mediated inflammatory responses in A $\beta 25$-35-stimulated microglia likely in part by decreasing the expression of

\section{TLR4 and RAGE.}

Because curcumin significantly inhibits HMGB1 production and HMGB1-mediated inflammatory responses in A 25 -35- stimulated microglia. We last to examine whether curcumin inhibits the toxicity of activated microglia to neurons. Our result showed that curcumin protected neurons against toxicity induced by A $\beta 25-35$-activated microglia, which had consistency with earlier studies $(24,25)$. Yang et al. indicated that curcumin could protect hippocampal 


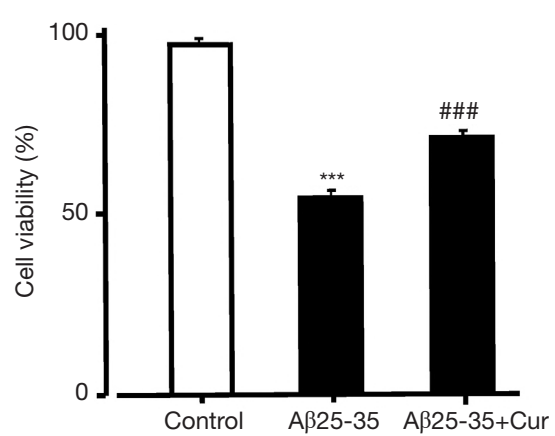

Figure 3 Effects of curcumin on the toxicity of A $\beta 25-35$-activated microglia to PC12 cells. PC12 cells were treated with conditioned medium from microglia treated with $\mathrm{A} \beta 25-35$ or A $\beta 25-35$ and curcumin. After $24 \mathrm{~h}$, the cell viability was examined by MTT assay. ***, $\mathrm{P}<0.001$, vs. control; \#\#\#, $\mathrm{P}<0.001$, vs. $\mathrm{A} \beta 25-35$. $\mathrm{PC} 12$ cells treated with $\mathrm{PBS}$ were set as control. All data were expressed as mean \pm standard deviation $(n=4)$. Cur, curcumin; $A \beta$, amyloid beta; PC12, Pheochromocytoma; PBS, phosphate buffer saline.

HT22 cells from toxicity mediated by erythrocyte lysistreated microglia (24). Guo et al. also found that curcumin suppressed the production of proinflammatory mediators in HIV-1-gp120-stimulated microglia and protected cortical neurons against HIV-1-induced toxicity (25).

In summary, the present study supplies evidence that curcumin could effectively inhibit neuroinflammation induced by A $\beta 25-35$ in primary rat microglia likely in part through suppressing the expression of HMGB1, TLR4, and RAGE. Our results add a new insight about the molecular mechanisms by which curcumin exerts anti-inflammatory effects against $\mathrm{A} \beta$-activated microglia.

\section{Acknowledgments}

Funding: The current study was supported by grants from the National Natural Science Foundation of China (grant no. 81271204), Natural Science Foundation of Zhejiang Province (grant no.LY17H090015), program from Science and Technology Bureau of Wenzhou (grant no. 2017Y0596).

\section{Footnote}

Conflicts of Interest: The authors have no conflicts of interest to declare.

Ethical Statement: The authors are accountable for all aspects of the work in ensuring that questions related to the accuracy or integrity of any part of the work are appropriately investigated and resolved. All animal experiments were conducted following the animal protocols approved by the Animal Ethics Committee of the Second Affiliated Hospital \& Yuying Children Hospital of Wenzhou Medical University.

\section{References}

1. Selkoe DJ. Alzheimer's disease results from the cerebral accumulation and cytotoxicity of amyloid beta-protein. J Alzheimers Dis 2001;3:75-80.

2. Wang MM, Miao D, Cao XP, et al. Innate immune activation in Alzheimer's disease. Ann Transl Med 2018;6:177.

3. Cai Z, Hussain MD, Yan LJ. Microglia, neuroinflammation, and beta-amyloid protein in Alzheimer's disease. Int J Neurosci 2014;124:307-21.

4. Spangenberg EE, Green KN. Inflammation in Alzheimer's disease: Lessons learned from microglia-depletion models. Brain Behav Immun 2017;61:1-11.

5. Scaffidi P, Misteli T, Bianchi ME. Release of chromatin protein HMGB1 by necrotic cells triggers inflammation. Nature 2002;418:191-5.

6. Gauley J, Pisetsky DS. The translocation of HMGB1 during cell activation and cell death. Autoimmunity 2009;42:299-301.

7. Arancio O, Zhang HP, Chen $\mathrm{X}$, et al. RAGE potentiates Abeta-induced perturbation of neuronal function in transgenic mice. Embo J 2004;23:4096-105.

8. Buchanan MM, Hutchinson M, Watkins LR, et al. Toll-like receptor 4 in CNS pathologies. J Neurochem 2010;114:13-27.

9. Mazarati A, Maroso M, Iori V, et al. High-mobility group box-1 impairs memory in mice through both toll-like receptor 4 and Receptor for Advanced Glycation End Products. Exp Neurol 2011;232:143-8.

10. Goozee KG, Shah TM, Sohrabi HR, et al. Examining the potential clinical value of curcumin in the prevention and diagnosis of Alzheimer's disease. Br J Nutr 2016;115:449-65.

11. Massa F, Meli R, Morbelli S, et al. Serum neurofilament light chain rate of change in Alzheimer's disease: potentials applications and notes of caution. Ann Transl Med 2019;7:S133.

12. Lim GP, Chu T, Yang F, et al. The curry spice curcumin reduces oxidative damage and amyloid pathology in an Alzheimer transgenic mouse. J Neurosci 2001;21:8370-7. 
13. Ye J, Zhang Y. Curcumin protects against intracellular amyloid toxicity in rat primary neurons. Int J Clin Exp Med 2012;5:44-9.

14. Gu Q, Guan H, Shi Q, et al. Curcumin attenuated acute Propionibacterium acnes-induced liver injury through inhibition of HMGB1 expression in mice. Int Immunopharmacol 2015;24:159-65.

15. Wang C, Nie H, Li K, et al. Curcumin inhibits HMGB1 releasing and attenuates concanavalin $\mathrm{A}$-induced hepatitis in mice. Eur J Pharmacol 2012;697:152-7.

16. Tu CT, Yao QY, Xu BL, et al. Curcumin Protects Against Concanavalin A-Induced Hepatitis in Mice Through Inhibiting the Cytoplasmic Translocation and Expression of High Mobility Group Box 1. Inflammation 2013;36:206-15.

17. Tu CT, Yao QY, Xu BL, et al. Protective effects of curcumin against hepatic fibrosis induced by carbon tetrachloride: Modulation of high-mobility group box 1 , Toll-like receptor 4 and 2 expression. Food Chem Toxicol 2012;50:3343-51.

18. Kim DC, Lee W, Bae JS. Vascular anti-inflammatory effects of curcumin on HMGB1-mediated responses in vitro. Inflamm Res 2011;60:1161-8.

19. Kim OS, Park EJ, Joe EH, et al. JAK-STAT signaling mediates gangliosides-induced inflammatory responses in

Cite this article as: He W, Yuan K, Ji B, Han Y, Li J. Protective effects of curcumin against neuroinflammation induced by $\mathrm{A} \beta 25-35$ in primary rat microglia: modulation of high-mobility group box 1, toll-like receptor 4 and receptor for advanced glycation end products expression. Ann Transl Med 2020;8(4):88. doi: 10.21037/atm.2019.12.147 brain microglial cells. J Biol Chem 2002;277:40594-601.

20. Qian X, Cao H, Ma Q, et al. Allopregnanolone attenuates A $\beta$ 25-35-induced neurotoxicity in PC12 cells by reducing oxidative stress. Int J Clin Exp Med 2015;8:13610-5.

21. Takata K, Kitamura Y, Kakimura J, et al. Role of high mobility group protein-1 (HMG1) in amyloid- $\beta$ homeostasis. Biochem Biophys Res Commun 2003;301:699-703.

22. Takata K, Kitamura Y, Tsuchiya D, et al. High Mobility Group Box Protein-1 Inhibits Microglial A $\beta$ Clearance and Enhances $\mathrm{A} \beta$ Neurotoxicity. J Neurosci Res 2004;78:880-91.

23. Takata K, Takada T, Ito A, et al. Microglial Amyloid- $\beta 1-40$ Phagocytosis Dysfunction Is Caused by High-Mobility Group Box Protein-1: Implications for the Pathological Progression of Alzheimer's Disease. Int J Alzheimers Dis 2012;2012:685739.

24. Yang Z, Zhao T, Zou Y, et al. Curcumin inhibits microglia inflammation and confers neuroprotection in intracerebral hemorrhage. Immunol Lett 2014;160:89-95.

25. Guo L, Xing Y, Pan R, et al. Curcumin protects microglia and primary rat cortical neurons against HIV-1 gp120mediated inflammation and apoptosis. PLoS One 2013;8:e70565. 